\title{
Alterations in Serum BDNF and GDNF Levels after 12 Weeks of Antidepressant Treatment in Female Outpatients with Major Depressive Disorder
}

\author{
Young-Min Park ${ }^{1}$ and Bun-Hee Lee ${ }^{2 凶}$ \\ ${ }^{1}$ Department of Psychiatry, Ilsan Paik Hospital, Inje University College of Medicine, Goyang, Republic of Korea \\ ${ }^{2}$ Maum \& Maum Psychiatric Clinic, Seoul, Republic of Korea
}

\begin{abstract}
Objective Some clinical studies have found alterations in the levels of serum brain-derived neurotrophic factor (BDNF) and glial cell line-derived neurotrophic factor (GDNF) after applying antidepressant treatment in patients with major depressive disorder (MDD). We evaluated the serum BDNF and GDNF levels before and after 12 weeks of antidepressant treatment in MDD outpatients.

Methods Serum BDNF and GDNF levels were measured in 23 female MDD outpatients at baseline and after 12 weeks of treatment. The severity of depression was measured with the Hamilton Depression Rating Scale-17 (HAMD-17). Remission of MDD to the treatment was defined as a posttreatment HAMD-17 score of $<7$.

Results Among MDD patients, 19 (82.6\%) subjects were in mild to moderate depression. The whole MDD patients had significantly higher serum BDNF and GDNF levels at baseline than those after 12 weeks of antidepressant treatment. The baseline serum BDNF and GDNF levels did not significantly between the remission and nonremission groups. The significant alteration in both BDNF and GDNF levels after antidepressant treatment were observed in patients with remission.

Conclusion The present study suggests that the baseline serum BDNF and GDNF levels are higher than the posttreatment levels in some mild-to-moderate MDD outpatients and the significant alteration in BDNF and GDNF level after treatment were observed in patients with remission.

Psychiatry Investig 2018;15(8):818-823
\end{abstract}

Key Words Brain-derived neurotrophic factor, Glial cell line-derived neurotrophic factor, Major depression, Remission.

\section{INTRODUCTION}

The neurotrophic hypothesis explains the pathogenesis of the development of major depression and the effect of antidepressant treatment on depression. ${ }^{1}$ Neurotrophic factors are a family of proteins responsible for the growth, survival, differentiation, and function of developing neurons and the maintenance of mature neurons, and they include brain-derived neurotrophic factor (BDNF) and glial cell line-derived neurotrophic factor (GDNF) ${ }^{2,3}$ Neurotrophic factors can play crucial roles in the formation and plasticity of neuronal

\footnotetext{
Received: December 31, 2017 Revised: March 12, 2018

Accepted: March 31, 2018

$\triangle$ Correspondence: Bun-Hee Lee, MD, PhD

Maum \& Maum Psychiatric Clinic, 399 Gosanja-ro, Dongdaemun-gu, Seoul 02566, Republic of Korea

Tel: +82-2-6959-0456, Fax: +82-2-6959-0457, E-mail: lee.bunhee@gmail.com

(a) This is an Open Access article distributed under the terms of the Creative Commons Attribution Non-Commercial License (http://creativecommons.org/licenses/by$\mathrm{nc} / 4.0$ ) which permits unrestricted non-commercial use, distribution, and reproduction in any medium, provided the original work is properly cited.
}

networks. ${ }^{4}$ The neurotrophic hypothesis of depression states that major depression can result from impaired neuronal plasticity in the brain, and treatment with antidepressant agents can promote variable forms of neuronal plasticity, which could be related to the response to antidepressants. ${ }^{1,4}$

BDNF is an important neurotrophic factor whose role in depression has been investigated in both animal and clinical studies. The results of animal studies suggest that chronic stress can decrease BDNF expression in the hippocampus, which can be alleviated by antidepressant treatment. ${ }^{1,5}$ Metaanalyses of clinical studies indicate that patients with major depressive disorder (MDD) have decreased serum and plasma BDNF levels, which can be increased by antidepressant treatment. ${ }^{6,7}$ An increase in the BDNF level after 6 or 8 weeks of antidepressant treatment was observed in responders but not in nonresponders to antidepressants among MDD patients. ${ }^{8,9}$ However, some animal and clinical studies have produced results that are inconsistent with the above findings. ${ }^{10,11}$ GDNF is expressed abundantly throughout the brain. ${ }^{12}$ It 
exerts neurotrophic effects on the development and maintenance of glial cells, and can influence the development, survival, and differentiation of dopaminergic, serotonergic, and GABAergic neurons. ${ }^{3}$ An animal study demonstrated that a neurotoxin insult enhanced GDNF expression, suggesting a neuroprotective effect of GDNF in the brain. ${ }^{13}$ In addition, clinical experiments have explored the GDNF levels in the peripheral blood, serum, and plasma of MDD patients. Although these studies have produced diverse findings, a metaanalytic study found that the serum GDNF level is lower in untreated MDD patients than in controls. ${ }^{14}$ However, postmortem and clinical studies have found that the GDNF level is increased in the parietal cortex of recurrent MDD patients and that the plasma GDNF level is increased in elderly MDD patients. ${ }^{15,16}$ An animal study found that exposure to chronic unpredictable stress activated astrocytes and increased GDNF levels in the hippocampus of mice. ${ }^{17}$

The neurotrophic hypothesis suggests that there is a deficit of serum BDNF and GDNF in MDD patients and that normal BDNF and GDNF levels can be restored by antidepressant treatment. However, clinical studies exploring serum BDNF and GDNF levels in MDD patients have produced conflicting findings. Clinical studies examining the effects of 12 and 24 weeks of treatment with several antidepressants in MDD patients have also produced mixed results. ${ }^{18-20}$ Treatment with escitalopram for 24 weeks did not change the serum BDNF level, ${ }^{20}$ while 12 weeks of treatment with mirtazapine or duloxetine significantly increased the serum or plasma BDNF level in MDD patients. ${ }^{18,19}$ However, one study found that an increase in the serum BDNF level was associated with treatment response, ${ }^{18}$ while another observed an increase in plasma BDNF in early nonresponders but not in early responders among MDD patients. ${ }^{19}$

The present study explored changes in serum BDNF and GDNF levels in MDD outpatients before and after 12 weeks of antidepressant treatment. We also compared serum BDNF and GDNF levels between remission and nonremission among MDD patients.

\section{METHODS}

\section{Subjects}

This study included 23 female consecutive outpatients from Ilsan Paik Hospital. These patients (age $=45.6 \pm 12.7$ years, mean $\pm S D$; age range $=19-65$ years) were diagnosed with MDD according to criteria of the Diagnostic and Statistical Manual of Mental Disorders-IV-text revision. Patients were excluded if they had any major mental disorders such as bipolar disorder, schizophrenia, or substance use disorder, or major medical and neurologic disorders. We also excluded subjects who were pregnant or lactating. The included subjects had been either medication-naïve or medication-free for at least 4 weeks prior to being assessed. Depressive and anxiety symptoms were assessed using the Hamilton Depression Rating Scale-17 (HAMD-17) 21,22 and the Hamilton Anxiety Rating Scale (HAMA) ${ }^{23}$ at baseline and 12 weeks after treatment. Then this study included MDD patients with 10 or more score of baseline HAMD-17 scale. The 23 included MDD patients comprised 10 who were experiencing their first episode and 13 who were experiencing a recurrent episode. In the recurrent MDD patients, their medication-free duration $44.9 \pm 76.0$ weeks (the range $=6$ weeks -6 years). The patients were treated with the following antidepressants with doses based on their psychiatric symptoms during this study: escitalopram $[\mathrm{n}=17,11.76 \pm 3.03 \mathrm{mg} /$ day (the range $=10-20$ $\mathrm{mg} /$ day) $]$, sertraline $[\mathrm{n}=4,75.0 \pm 28.9 \mathrm{mg} /$ day (the range $=50-$ $100 \mathrm{mg} /$ day)], paroxetine ( $\mathrm{n}=1,25 \mathrm{mg} /$ day), or duloxetine $(\mathrm{n}=1$, $60 \mathrm{mg} /$ day).

This study did allow the limited concomitant pharmacotherapy. We prescribed alprazolam $(0.25 \mathrm{mg}$ to $1.0 \mathrm{mg}$ per day) for anxiety and clonazepam ( $0.25 \mathrm{mg}$ or $0.5 \mathrm{mg}$ per day) for sleep disturbance, and their doses were altered according to the severity of anxiety or sleep disturbance.

The study protocol was approved by the Ethics Committee of Ilsan Paik Hospital, Inje University College of Medicine (IRB No. 2011-11-314), and written informed consent was obtained from all of the subjects when they entered the study.

\section{Measurements of serum BDNF and GDNF levels}

Blood samples for measuring the BDNF and GDNF levels were taken between 8 a.m. and 9 a.m. following an overnight fast and centrifuged to separate the serum. The serum BDNF and GDNF levels were measured at baseline and after 12 weeks of treatment.

Serum BDNF and GDNF levels were analyzed using enzyme-linked immunosorbent assay (ELISA) kits (Human Magnetic Luminex Screening Assay, R\&D Systems, Minneapolis, MN, USA). Each assay was performed in duplicate. The actual concentration of each sample was calculated using the seven-parameter-fit logistic-curve equation. The ELISA plate readings were conducted using a microplate reader (VersaMax, Molecular Devices, Sunnyvale, CA, USA).

\section{Statistical analysis}

The Shapiro-Wilk test revealed that the serum BDNF levels $(\mathrm{W}=0.981, \mathrm{p}=0.927)$ and GDNF levels $(\mathrm{W}=0.892, \mathrm{p}=0.058)$ were normally distributed. The demographic data, clinical variables, and serum BDNF and GDNF levels were compared between two groups using Student's t-tests and chisquare tests. Serum BDNF and GDNF levels were compared 
among the four antidepressant agents using Kruskal-Wallis test. Pearson's correlation coefficients were calculated to examine the relationships between serum BDNF or GDNF levels and the analyzed clinical variables. The pretreatment and posttreatment BDNF levels, GDNF levels, and HAMD-17 and HAMA scores in MDD patients were compared by paired t-tests. Remission of MDD to the treatment was defined as a posttreatment HAMD-17 score of $<7 .{ }^{24}$ The null hypothesis was rejected at $\mathrm{p}<0.05$. The statistical analysis was conducted using the SALT 2.5 (Istech, Goyang, Korea) software packages.

\section{RESULTS}

The HAMD-17 and HAMA scores were 18.1 \pm 5.6 and $19.9 \pm 6.3$, respectively, at baseline. According to the baseline HAMD-17 scores, 8 (34.8\%) patients were in mild depression scores (10-16 of HAMD-17 score), 11 (47.8\%) in moderate depression (17-23 of HAMD-17 score), and 4 (17.4\%) in severe depression ( $\geq 24$ of HAMD-17 score). And the HAMD-17 and HAMA scores were decreased significantly to $8.8 \pm 6.3(\mathrm{t}=6.544, \mathrm{p}<0.001)$ and 11.0 $\pm 7.4(\mathrm{t}=5.777, \mathrm{p}<0.001)$ after 12 weeks of antidepressant treatment (Table 1).

The baseline serum BDNF and GDNF levels were 18.15 \pm $8.37 \mathrm{ng} / \mathrm{mL}$ and $1.84 \pm 0.94 \mathrm{pg} / \mathrm{mL}$, respectively. There was no significant correlation between serum BDNF level and age $(r=0.038, p=0.863)$ among all of the subjects, whereas the serum GDNF level was significantly correlated with age $(r=$ -0.541, $\mathrm{p}=0.008$ ). The serum BDNF level was not correlated with the HAMD-17 score $(\mathrm{r}=0.042, \mathrm{p}=0.849)$ or the HAMA score $(r=-0.066, p=0.765)$. The serum GDNF level was not correlated with the HAMD-17 score $(\mathrm{r}=-0.296, \mathrm{p}=0.170)$ but it was correlated with the HAMA score $(\mathrm{r}=-0.423, \mathrm{p}=0.044)$; however, this correlation was not significant after controlling for age as a covariate $(\mathrm{r}=-0.253, \mathrm{p}=0.255)$. There were no significant differences in serum BDNF $(\mathrm{t}=-0.743, \mathrm{p}=0.466)$ and GDNF levels ( $\mathrm{t}=0.125, \mathrm{p}=0.902)$ between the first-episode and recurrent-episode patients.

The 12-week posttreatment serum BDNF and GDNF levels were significantly decreased in comparison with the baseline levels: $12.58 \pm 3.88 \mathrm{ng} / \mathrm{mL}(\mathrm{t}=2.748, \mathrm{p}=0.012)$ and $1.17 \pm 0.71 \mathrm{pg} / \mathrm{mL}(\mathrm{t}=3.430, \mathrm{p}=0.003)$, respectively (Table 1$)$. There were no significant differences in posttreatment serum BDNF $\left(\chi^{2}=3.274, p=0.351\right)$ and GDNF levels $\left(\chi^{2}=2.722\right.$, $\mathrm{p}=0.436$ ) among the four antidepressants.

Ten subjects of the all MDD patients (43.5\%) were in remission while the others (56.5\%) were in nonremission after 12 weeks of antidepressant treatment, based on a cutoff total HAMD-17 score of $<7$ for remission. The demographic data and clinical variables between remission and nonremission patients were presented in Table 2. The baseline BDNF and GDNF levels were higher in the remission patients (20.31 $10.10 \mathrm{ng} / \mathrm{mL}$ and $2.10 \pm 0.92 \mathrm{pg} / \mathrm{mL}$, respectively) than in the nonremission patients $(16.50 \pm 6.71 \mathrm{ng} / \mathrm{mL}$ and $1.63 \pm 0.95$ $\mathrm{pg} / \mathrm{mL}$ ), but the differences were not statistically significant $(\mathrm{t}=-1.088, \mathrm{p}=0.289$, and $\mathrm{t}=-1.209, \mathrm{p}=0.240)$. The posttreatment serum BDNF and GDNF levels did not differ significantly between remission patients and nonremission patients

Table 1. Differences in variables between baseline and 12-week posttreatment in outpatients with major depressive disorder

\begin{tabular}{|c|c|c|c|}
\hline & Baseline & After 12-week & Statistics* \\
\hline HAMD-17 & $18.1 \pm 5.6$ & $8.8 \pm 6.3$ & $\mathrm{t}=6.544, \mathrm{p}<0.001$ \\
\hline HAMA & $19.9 \pm 6.3$ & $11.0 \pm 7.4$ & $\mathrm{t}=5.777, \mathrm{p}<0.001$ \\
\hline Serum BDNF (ng/mL) & $18.15 \pm 8.37$ & $12.58 \pm 3.88$ & $\mathrm{t}=2.748, \mathrm{p}=0.012$ \\
\hline Serum GDNF (pg/mL) & $1.84 \pm 0.94$ & $1.17 \pm 0.71$ & $\mathrm{t}=3.430, \mathrm{p}=0.003$ \\
\hline
\end{tabular}

*the baseline and 12-week posttreatment HAMD and HAMA scores, BDNF levels, and GDNF levels in patients with major depressive disorder were compared by paired t-tests. HAMD-17: Hamilton Depression Rating Scale-17, HAMA: Hamilton Anxiety Rating Scale, BDNF: brain-derived neurotrophic factor, GDNF: glial cell line-derived neurotrophic factor

Table 2. Comparison of the demographic data and clinical variables between MDD patient with remission and with nonremission

\begin{tabular}{|c|c|c|c|}
\hline & $\begin{array}{l}\text { MDD patients with remission } \\
\qquad(\mathrm{N}=10,43.5 \%)\end{array}$ & $\begin{array}{l}\text { MDD patients with nonremission } \\
\qquad(\mathrm{N}=13,56.5 \%)\end{array}$ & Statistics \\
\hline Age (years) & $43.0 \pm 10.6$ & $47.5 \pm 14.3$ & $\mathrm{t}=0.841, \mathrm{p}=0.410$ \\
\hline Recurrent episode, N (\%) & $7(70)$ & $6(46.2)$ & $\chi^{2}=1.308, \mathrm{p}=0.253$ \\
\hline Duration of depression (months) & $3.3 \pm 0.5$ & $3.1 \pm 0.3$ & $\mathrm{t}=-1.304, \mathrm{p}=0.214$ \\
\hline HAMD-17 & $17.50 \pm 5.38$ & $18.62 \pm 5.94$ & $\mathrm{t}=0.465, \mathrm{p}=0.647$ \\
\hline HAMA & $19.10 \pm 5.34$ & $20.62 \pm 7.03$ & $\mathrm{t}=0.566, \mathrm{p}=0.577$ \\
\hline
\end{tabular}

HAMD-17: Hamilton Depression Rating Scale-17, HAMA: Hamilton Anxiety Rating Scale, MDD: major depressive disorder 
$(\mathrm{t}=0.905, \mathrm{p}=0.377 ; \mathrm{t}=0.153, \mathrm{p}=0.880$, respectively) (Table 3 ). The BDNF levels were significantly changed from baseline to 12 -week of treatment in patients with remission $(\mathrm{t}=2.322$, $\mathrm{p}=0.045)$, not those with nonremission $(\mathrm{t}=1.505, \mathrm{p}=0.163)$. However, the GDNF levels were significantly decreased both in patients with remission $(\mathrm{t}=2.389, \mathrm{p}=0.041)$ and nonremission $(\mathrm{t}=2.833, \mathrm{p}=0.018)$.

\section{DISCUSSION}

The present study found that the serum BDNF and GDNF levels in MDD patients were significantly decreased after 12 weeks of treatment with antidepressants. In other words, the serum BDNF and GDNF levels were significantly higher at baseline than after 12 weeks of treatment.

Many clinical studies and meta-analyses have found that the serum and plasma BDNF levels measured before antidepressant treatment in MDD patients are lower than those in healthy controls, with the BDNF levels in MDD patients increasing or normalizing after antidepressant treatment. . $^{-9,25,26}$ However, other previous studies found that the pretreatment serum BDNF levels in MDD patients were higher than those in healthy controls. ${ }^{10,27,28}$ Elfving et al. ${ }^{10}$ compared serum BDNF levels and Val66Met polymorphisms between 162 MDD outpatients and 289 healthy controls, and found that the pretreatment serum BDNF levels were increased in MDD patients in comparison with healthy controls. Kheirouri et al. ${ }^{27}$ also observed that the serum BDNF levels were significantly higher in $44 \mathrm{MDD}$ patients than in 42 healthy controls. We also found that the pretreatment serum BDNF level was significantly higher than the posttreatment BDNF level.

These inconsistent findings from clinical studies of higher or lower pretreatment serum BDNF levels in MDD patients compared with healthy controls seem to be associated with variations in recurrence (first episode or recurrent episode), severity, duration, and case type (inpatient or outpatient). In the study of Elfving et al. ${ }^{10}$ the MDD patients were outpatients and consisted of $83 \%$ with mild-to-moderate MDD and $17 \%$ with severe MDD. In the study of Kheirouri et al. ${ }^{27}$ the MDD subjects were also outpatients, they were in their first MDD episode, and their mean HAMD-17 score was 17.8. The present study also included MDD outpatients whose mean HAMD-17 score at baseline was 18.1. Among MDD patients in the present study, 19 (82.6\%) subjects were in mild to moderate depression. However, Yoshimura et al. ${ }^{8}$ and Lee et al. ${ }^{26}$ found a lower serum or plasma BDNF level in MDD patients compared with healthy controls, and showed mean HAMD-17 scores in MDD patients of 24 and 27.7, respectively. Together these findings suggest that some clinical characteristics of MDD patients-such as mild-to-moderate severity of depression or being outpatients-appear to be associated with the increased BDNF level during the development of major depression.

While many animal studies have found reduced BDNF levels in depression models, there have been several animal experiments producing the opposite result, which could be explained by an association with depression and anxiety behavior. ${ }^{11}$ One animal study suggested that changes in BDNF level or brain neuroplasticity could differ according to the vulnerability to depression or the resilience to stress. ${ }^{29}$ In addition, other animal studies found increased BDNF levels in the serum and hippocampus in two depression models with olfactory bulbectomy and chronic unpredictable stress. ${ }^{30,31}$ It can therefore be speculated that exposure to certain stressors activates brain neuroplasticity resulting in enhanced BDNF, and hence such an increase in BDNF might reflect an adaptive protective response to stressors or a stress-related compensating process for homeostasis. In addition, some investigators claim that the current BDNF hypothesis is too simplistic, and they suggest that neuroplastic responses to stress and antidepressants are involved in the changes in

Table 3. Differences in baseline and 12-week posttreatment serum BDNF and GDNF levels between MDD patient with remission and with nonremission

\begin{tabular}{|c|c|c|c|}
\hline & $\begin{array}{l}\text { MDD patients with remission } \\
(\mathrm{N}=10,43.5 \%)\end{array}$ & $\begin{array}{l}\text { MDD patients with nonremission } \\
\qquad(\mathrm{N}=13,56.5 \%)\end{array}$ & Statistics* \\
\hline Baseline BDNF (ng/mL) & $20.31 \pm 10.10$ & $16.50 \pm 6.71$ & $\mathrm{t}=-1.088, \mathrm{p}=0.289$ \\
\hline Posttreatment BDNF (ng/mL) & $11.95 \pm 4.13$ & $12.80 \pm 3.91$ & $\mathrm{t}=0.905, \mathrm{p}=0.377$ \\
\hline Statistics ${ }^{\dagger}$ & $\mathrm{t}=2.322, \mathrm{p}=0.045$ & $\mathrm{t}=1.505, \mathrm{p}=0.163$ & \\
\hline Baseline GDNF (pg/mL) & $2.10 \pm 0.92$ & $1.63 \pm 0.95$ & $\mathrm{t}=-1.209, \mathrm{p}=0.240$ \\
\hline Posttreatment GDNF (pg/mL) & $1.17 \pm 0.78$ & $1.12 \pm 0.62$ & $\mathrm{t}=0.153, \mathrm{p}=0.880$ \\
\hline Statistics ${ }^{\dagger}$ & $\mathrm{t}=2.389, \mathrm{p}=0.041$ & $\mathrm{t}=2.833, \mathrm{p}=0.018$ & \\
\hline
\end{tabular}

*the serum BDNF and GDNF levels were compared between patients with remission and with nonremission by t-tests, ${ }^{\dagger}$ the baseline and 12 week posttreatment BDNF and GDNF levels in MDD patients were compared by paired t-tests. BDNF: brain-derived neurotrophic factor, GDNF: glial cell line-derived neurotrophic factor, MDD: major depressive disorder 
BDNF levels, with these effects being both region-specific and antidepressant-specific, while neuroplastic resilience or antidepressant responses act according to the background of potent genetic and environmental modifiers. ${ }^{32}$

We also found that the serum GDNF level in MDD patients changed between baseline and after 12 weeks of antidepressant treatment. Moreover, these significant decreases in BDNF and GDNF level after the treatment was observed in patients with remission. One clinical study explored the relationship between the serum BDNF level and HAMD-17 score in $25 \mathrm{MDD}$ patients during 6 weeks of antidepressant treatment, and found that a higher pretreatment level of serum $\mathrm{BDNF}$ was associated with a treatment response, $\mathrm{a} \geq 50 \%$ reduction in the HAMD-17 score, and remission at week $6 .^{33}$ However, previous studies have produced inconsistent findings about alterations in serum or plasma GDNF levels after antidepressant treatment. Some studies showed that serum GDNF levels in MDD patients increased significantly after applying several types of antidepressant treatment, including pharmacologic or nonpharmacologic therapies. ${ }^{34-36}$ Increases in serum GDNF levels were also observed in treatment responders or remitters-but not in nonresponders-among MDD patients. ${ }^{34,35}$

However, other studies found that plasma GDNF levels in MDD patients did not change significantly after 6 weeks of antidepressant treatment. ${ }^{37,38}$ Furthermore, the present study found a reduction in serum GDNF levels in MDD patients after 12 weeks of antidepressant treatment, and that both the baseline GDNF and BDNF levels were higher in the remission patients than in the nonremission patients. Wang et al. ${ }^{16}$ reported that plasma GDNF levels were higher in elderly MDD patients than in healthy controls. They also observed that the increase in the GDNF level was positively correlated with a better cognitive performance, suggesting that a high GDNF level is a protective response for cognitive function in elderly MDD patients. ${ }^{16}$ There have also been animal studies demonstrating that GDNF can exert neuroprotective effects in the brain and that GDNF expression in the brain increases to cope with a neurotoxin insult. ${ }^{13,39}$ Together these findings indicate that increased GDNF as a compensatory response might appear in some MDD subjects, which could explain our finding of MDD patients having a higher serum GDNF level before antidepressant treatment and being able to reach remission after treatment.

This study was subject to several limitations: 1 ) the sample was relatively small, it comprised only female patients, and it did not include healthy controls, 2) four different kinds of antidepressant agents were administered, and 3) the serum BDNF and GDNF levels were measured at only two time points (at baseline and after 12 weeks of antidepressant treat- ment). Previous reports suggest that gender can influence serum or plasma BDNF levels. ${ }^{8,9,40}$ However, we did not recruit enough sample size of male MDD patients, and finally we had excluded male patients from this analysis. It will require further studies with larger subjects with male and female patients. This study showed that there were no significant differences in posttreatment BDNF and GDNF levels among the four antidepressants. Moreover, the previous clinical studies have suggested that the significant alterations in serum or plasma BDNF levels were observed in MDD patients with clinical response to antidepressant treatments. ${ }^{8,9,41,42}$ Mikoteit et al..$^{33}$ reported that the serum BDNF level increased significantly from baseline to after 2 weeks of treatment and then subsequently decreased again after 6 weeks of treatment. This implies that serum levels should be examined at several times points during treatments with antidepressant.

The results of this study suggest that the baseline serum BDNF and GDNF levels are higher than the posttreatment levels in some mild-to-moderate MDD outpatients. In addition, the significant alteration in BDNF and GDNF level after antidepressant treatment were observed in patients with remission. Such increases in serum BDNF and GDNF levels in major depression could reflect an adaptive protective or compensating response to certain stressors in brain neuroplasticity in mild-to-moderate major depression outpatients.

\section{Acknowledgments}

This work was supported by a grant from the National Research Foundation of Korea (NRF), funded by the Ministry of Education (NRF2014R1A1A2A10059345).

\section{REFERENCES}

1. Castrén E, Võikar V, Rantamäki T. Role of neurotrophic factors in depression. Curr Opin Pharmacol 2007;7:18-21.

2. Warner-Schmidt JL, Duman RS. Hippocampal neurogenesis: opposing effects of stress and antidepressant treatment. Hippocampus 2006;16:239-249.

3. Ducray A, Krebs SH, Schaller B, Seiler RW, Meyer M, Widmer HR. GDNF family ligands display distinct action profiles on cultured GABAergic and serotonergic neurons of rat ventral mesencephalon. Brain Res 2006;1069:104-112.

4. Pittenger C, Duman RS. Stress, depression, and neuroplasticity: a convergence of mechanisms. Neuropsychopharmacology 2008;33:88-109.

5. Tsankova NM, Berton O, Renthal W, Kumar A, Neve RL, Nestler EJ. Sustained hippocampal chromatin regulation in a mouse model of depression and antidepressant action. Nat Neurosci 2006;9:519-525.

6. Sen S, Duman R, Sanacora G. Serum brain-derived neurotrophic factor, depression, and antidepressant medications: meta-analyses and implications. Biol Psychiatry 2008;64:527-532.

7. Molendijk ML, Spinhoven P, Polak M, Bus BA, Penninx BW, Elzinga BM. Serum BDNF concentrations as peripheral manifestations of depression: evidence from a systematic review and meta-analyses on 179 associations ( $\mathrm{n}=9484)$. Mol Psychiatry 2014;19:791-800.

8. Yoshimura R, Mitoma M, Sugita A, Hori H, Okamoto T, Umene W, et al. Effects of paroxetine or milnacipran on serum brain-derived neurotrophic factor in depressed patients. Prog Neuropsychopharmacol 
Biol Psychiatry 2007;31:1034-1037.

9. Lee HY, Kim YK. Plasma brain-derived neurotrophic factor as a peripheral marker for the action mechanism of antidepressants. Neuropsychobiology 2008;57:194-199.

10. Elfving B, Buttenschøn HN, Foldager L, Poulsen PH, Andersen JH, Grynderup MB, et al. Depression, the Val66Met polymorphism, age, and gender influence the serum BDNF level. J Psychiatr Res 2012;46: 1118-1125.

11. Groves JO. Is it time to reassess the BDNF hypothesis of depression? Mol Psychiatry 2007;12:1079-1088.

12. Airaksinen MS, Saarma M. The GDNF family: signalling, biological functions and therapeutic value. Nat Rev Neurosci 2002;3:383-394.

13. Tang YP, Ma YL, Chao CC, Chen KY, Lee EH. Enhanced glial cell linederived neurotrophic factor mRNA expression upon (-)-deprenyl and melatonin treatments. J Neurosci Res 1998;53:593-604.

14. Lin PY, Tseng PT. Decreased glial cell line-derived neurotrophic factor levels in patients with depression: a meta-analytic study. J Psychiatr Res 2015;63:20-27.

15. Michel TM, Frangou S, Camara S, Thiemeyer D, Jecel J, Tatschner T, et al. Altered glial cell line-derived neurotrophic factor (GDNF) concentrations in the brain of patients with depressive disorder: a comparative post-mortem study. Eur Psychiatry 2008;23:413-420.

16. Wang X, Hou Z, Yuan Y, Hou G, Liu Y, Li H, et al. Association study between plasma GDNF and cognitive function in late-onset depression. J Affect Disord 2011;132:418-421.

17. Bian Y, Pan Z, Hou Z, Huang C, Li W, Zhao B. Learning, memory, and glial cell changes following recovery from chronic unpredictable stress. Brain Res Bull 2012;88:471-476.

18. Gupta R, Gupta K, Tripathi AK, Bhatia MS, Gupta LK. Effect of mirtazapine treatment on serum levels of brain-derived neurotrophic factor and tumor necrosis factor- $\alpha$ in patients of major depressive disorder with severe depression. Pharmacology 2016;97:184-188.

19. Fornaro M, Escelsior A, Rocchi G, Conio B, Magioncalda P, Marozzi V, et al. BDNF plasma levels variations in major depressed patients receiving duloxetine. Neurol Sci 2015;36:729-734.

20. Ladea M, Bran M. Brain derived neurotrophic factor (BDNF) levels in depressed women treated with open-label escitalopram. Psychiatr Danub 2013;25:128-132.

21. Hamilton M. A rating scale for depression. J Neurol Neurosurg Psychiatry 1960;23:56-62.

22. Zimmerman M, Martinez JH, Young D, Chelminski I, Dalrymple K. Severity classification on the Hamilton Depression Rating Scale. J Affect Disord 2013;150:384-388.

23. Maier W, Buller R, Philipp M, Heuser I. The Hamilton Anxiety Scale: reliability, validity and sensitivity to change in anxiety and depressive disorders. J Affect Disord 1988;14:61-68.

24. Riedel M, Moller HJ, Obermeier M, Schennach-Wolff R, Bauer M, Adli $\mathrm{M}$, et al. Response and remission criteria in major depression--a validation of current practice. J Psychiatr Res 2010;44:1063-1068.

25. Polyakova M, Stuke K, Schuemberg K, Mueller K, Schoenknecht P, Schroeter ML. BDNF as a biomarker for successful treatment of mood disorders: a systematic \& quantitative meta-analysis. J Affect Disord 2015;174:432-440.

26. Lee BH, Kim H, Park SH, Kim YK. Decreased plasma BDNF level in depressive patients. J Affect Disord 2007;101:239-244.

27. Kheirouri S, Noorazar SG, Alizadeh M, Dana-Alamdari L. Elevated brain-derived neurotrophic factor correlates negatively with severity and duration of major depressive episodes. Cogn Behav Neurol 2016; 29:24-31.

28. Papakostas GI, Shelton RC, Kinrys G, Henry ME, Bakow BR, Lipkin $\mathrm{SH}$, et al. Assessment of a multi-assay, serum-based biological diagnostic test for major depressive disorder: a pilot and replication study. Mol Psychiatry 2013;18:332-339.

29. Blugeot A, Rivat C, Bouvier E, Molet J, Mouchard A, Zeau B, et al. Vulnerability to depression: from brain neuroplasticity to identification of biomarkers. J Neurosci 2011;31:12889-12899.

30. Luo KR, Hong CJ, Liou YJ, Hou SJ, Huang YH, Tsai SJ. Differential regulation of neurotrophin S100B and BDNF in two rat models of depression. Prog Neuropsychopharmacol Biol Psychiatry 2010;34:1433-1439.

31. Larsen MH, Mikkelsen JD, Hay-Schmidt A, Sandi C. Regulation of brain-derived neurotrophic factor (BDNF) in the chronic unpredictable stress rat model and the effects of chronic antidepressant treatment. J Psychiatr Res 2010;44:808-816.

32. Krishnan V, Nestler EJ. The molecular neurobiology of depression. Nature 2008;455:894-902.

33. Mikoteit T, Beck J, Eckert A, Hemmeter U, Brand S, Bischof R, et al. High baseline BDNF serum levels and early psychopathological improvement are predictive of treatment outcome in major depression. Psychopharmacology (Berl) 2014;231:2955-2965.

34. Zhang X, Zhang Z, Xie C, Xi G, Zhou H, Zhang Y, et al. Effect of treatment on serum glial cell line-derived neurotrophic factor in depressed patients. Prog Neuropsychopharmacol Biol Psychiatry 2008;32:886890.

35. Zhang X, Zhang Z, Sha W, Xie C, Xi G, Zhou H, et al. Electroconvulsive therapy increases glial cell-line derived neurotrophic factor (GDNF) serum levels in patients with drug-resistant depression. Psychiatry Res 2009;170:273-275.

36. Sun H, Zhao H, Ma C, Bao F, Zhang J, Wang DH, et al. Effects of electroacupuncture on depression and the production of glial cell line-derived neurotrophic factor compared with fluoxetine: a randomized controlled pilot study. J Altern Complement Med 2013;19:733-739.

37. Brunoni AR, Machado-Vieira R, Zarate CAJ, Vieira EL, Valiengo L, Benseñor IM, et al. Assessment of non-BDNF neurotrophins and GDNF levels after depression treatment with sertraline and transcranial direct current stimulation in a factorial, randomized, sham-controlled trial (SELECT-TDCS): an exploratory analysis. Prog Neuropsychopharmacol Biol Psychiatry 2015;56:91-96.

38. Lee BH, Hong JP, Hwang JA, Na KS, Kim WJ, Trigo J, et al. Plasma glial cell line-derived neurotrophic factor in patients with major depressive disorder: a preliminary study. Acta Neuropsychiatr 2016;28:45-50.

39. Cheng H, Fu YS, Guo JW. Ability of GDNF to diminish free radical production leads to protection against kainate-induced excitotoxicity in hippocampus. Hippocampus 2004;14:77-86.

40. Choi SW, Bhang S, Ahn JH. Diurnal variation and gender differences of plasma brain-derived neurotrophic factor in healthy human subjects. Psychiatry Res 2011;186:427-430.

41. Lee BH, Myint AM, Kim YK. Psychotropic drugs on in vitro brain-derived neurotrophic factor production in whole blood cell cultures from healthy subjects. J Clin Psychopharmacol 2010;30:623-627.

42. Lee BH, Kim YK. The roles of BDNF in the pathophysiology of major depression and in antidepressant treatment. Psychiatry Investig 2010;7:231-235. 